\title{
Topographical Pattern Analysis Using Wavelet Based Coherence Connectivity Estimation in the Distinction of Meditation and Non-meditation EEG
}

\author{
Laxmi Shaw \\ Department of Electrical Engineering \\ Indian Institute of Technology Kharagpur \\ Kharagpur, India-721302 \\ Email: laxmishaw1983@gmail.com
}

\author{
Aurobinda Routray \\ Department of Electrical Engineering \\ Indian Institute of Technology Kharagpur \\ Kharagpur, India-721302 \\ Email: aurobinda.routray@gmail.com
}

\begin{abstract}
Classification of EEG signal involved in a particular cognitive activity has found many application in brain-computer interface (BCI). In specific, use of classification algorithms to highly multivariate non-stationary recordings like EEG is a challenging and promising task. This study investigated two substantial novelty of the topics, (1) Distinction between meditation (Kriya Yoga) and non-meditation state allied EEG, (2) Characterization of the underlying mechanism of cognitive process that is associated with meditation using topographical analysis. The topographic wavelet coherence based brain connectivity between two different groups is shown. Two groups of data, one with 23 meditators (meditator group) and other with ten non-meditators (controlled group) are analyzed. The spatial distribution between two groups can be well distinguished by the topographical approach. The quantification has been done by the colour intensity embedded in the topographical plots. The wavelet coherence is found to be a different parameter to represent the distinctiveness between two groups. The time-frequency quantification regarding wavelet coherence spectrum is shown the unique patterns among meditators and non-meditators. Thus time-frequency based wavelet coherence has found to be an unusual brain pattern in the distinction between meditators and non-meditators.
\end{abstract}

\section{INTRODUCTION}

Amongst different neuroimaging methods, Electroencephalographic (EEG) and Magneto-encephalographic (MEG) signals are preferred to capture the direct reflection of neural firing in the brain, exhibiting a high temporal resolution (in milliseconds) despite a low spatial resolution (of the order of a few square centimetres) [1]. The brain activity can be captured in many ways, among them, EEG is the non-invasive way of acquiring the brain signal. Any cognitive activity is being reflected in distinctive brain waves [2]. Among different cognitive activity, meditation is raised as a fascinating topic among the different study of neurophysiological behavior, $\operatorname{cog}$ nition, and consciousness. The meditation and their benefits have grabbed the attention of 'society of human neuroscience' because of many advantages to reducing stress related illness of today's fast living life style [3], [4]. Unlike meditation, any cognitive activities have their explicit indication in the human brain over EEG [2].
The challenging problem in brain connectomic studies is the classification of brain connectivity coming from more or less similar cognitive activity [5]. However, it's relatively difficult task to interpret the EEG waves by only looking at its dynamicity [6]. To visualize the inherent neural dynamics during meditation and resting brain state, both time and frequency behavior have considered in this study. We have depicted wavelet coherence connectivity study represented in cuttingedge topographical patterns [7]. The topographic approach has taken into consideration as a case study scheme in the distinction of meditation and non-meditation state-aided with EEG.

The topographical EEG analysis is the representation of the Spatio-temporal distribution of magnitude of the corresponding parameter. It has specified for three reasons: (1) ease of visual interpretation in the spatial domain, (2) ROI based inspection and (3) conformity inference using color bar representation throughout [8]. The functional brain connectivity cortico-cortical interactions can easily be interpreted by the topography. The brain topography is the descriptive analysis of characterizing the interrelationships of cortical areas concerning the functional connectivity. In brain connectivity study, fallacies often originate due to the cross talk between EEG channels and sensor space, termed as volume conduction effect [9]. It is the effects of recording electrical potential at a distance from their source generator. Since the recording electrodes are not in direct contact with the muscles or nerve, there is a medium which separates the two. In Practice, volume conduction found in almost all neurophysiological recordings.

In the existing literature, there are various classification techniques present to classify the EEG patterns into two or more than two groups involved in a different cognitive activity (In our case is two groups (meditators and nonmeditators)). Most of the classification algorithms work on the brain features. Sometimes it's hard to extract features from the EEG signals because of its large data dimensionality, nonlinearity, and non-stationarity. In multichannel EEG, the coherence between channels also can be featured. Assuming this, the wavelet coherence which preserves the time-frequency 
information between the two or more time series has been considered. A few literature has addressed the use of topographical approach for neurophysiological classification problems. In [10], the connectivity through spectral coherence is utilized for the biometric signature for the automatic people recognition [11]. It has discussed, instead of extracting different temporal features from the EEG signal, the coupling of different brain regions also possess some unique signature pattern within itself. Some of the literature also presented the use of wavelet coherence estimation in the Chinese Stroop task [12] study. One more work has debated the coupling between neural signal using wavelet-based coherence estimation in [13]. The use of the topographical distinction between meditators and non-meditator groups and their classification using waveletbased coherence has very rarely discussed.

The remaining section of the paper is organized as follows. Section II presents the methodological details of the data acquisition and preprocessing techniques. It also discusses the mathematical detailing of the concept of the wavelet coherence connectivity and their utility in the functional brain neural path estimation. Eventually, Section III shows the results in terms of topographical presentation and non-parametric comparison between meditators and non-meditators in average subjects. Section IV mentions the significance of the obtained results in the discussion section and finally, the paper concludes in Section V.

\section{METHODS}

\section{A. Experimentation, Proposed framework and ROI Selection}

The present study directly follows from the EEG experiments and the preprocessing techniques published in [14], [15] based on the same study protocol and method. For the sake of completeness, a short description of the experimentation and data collection is described below. Fig. 1 illustrates the overall framework for the wavelet-based coherence connectivity analysis in EEG data and their common interpretation concerns with meditative state and non-meditative state associated EEG. The selection of ROI is depicted considering the symmetry position of the electrodes and indicating the major brain lobes. The major brain lobes are shown in Fig.2 and the selected EEG electrodes are Fp1, Fp2, F3, F4, Fz, F7, F8, C3, C4, Cz, T7, T8, P3, P4, Pz, P7, P8, O1, and O2.

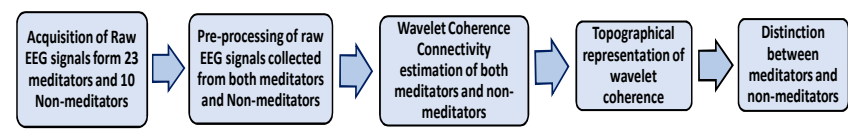

Fig. 1. Pipeline of the proposed approach for the distinction between meditators and non-meditators

\section{B. EEG Recordings and Data Preprocessing}

The EEG data were collected from 23 meditators and ten non-meditators (control group). The EEG data have been collected from 64 channels RMS Victa EEG machine, at a

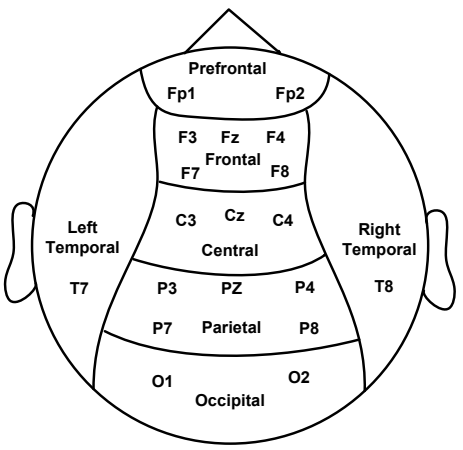

Fig. 2. Different brain lobes and selected 19 channels considered for wavelet coherence estimation

sampling rate of $256 \mathrm{~Hz}$ and 16-bit resolution. The data has been acquired for the duration of approximately 9 minutes data. However, nearly $4.17 \mathrm{mins}$ data has been used for the wavelet-based coherence study. Furthermore, the raw data has to be preprocessed for further wavelet based coherence analysis to remove unwanted noises and artifact. The pre-processing steps directly follow the steps mentioned in [14], [15]. The study protocol follows the same preprocessing techniques for the further analysis.

\section{Wavelet coherence (WC)}

To some extent, the problem due to non-stationarity has been overcome by wavelet analysis. Since the wavelet analysis preserves both time and frequency information, it measures the functional interrelations between the pair of signals. In this case, it is obtained from neuro-cortical regions of two or more EEG electrodes [5], [13]. In contrast to Fourier analysis, wavelet analysis has been developed to analyze signals with rapidly changing spectra [16]. It estimates the spectral characteristics of the signal as a function of time. Furthermore, the wavelet coherence is mostly used to detect common time-localized oscillation in non-stationary signals such as EEG. In the situation where EEG recordings were captured during meditation.

In some sense, the wavelet coherence that has the same form as the Fourier-based coherence function, namely the ratio of the cross spectrum to the product of the auto-spectrum of two signals $x$ and $y$ [17]. The mathematical details of the measure of wavelet coherence are mentioned below:-

Let $x$ and $y$ represent the two EEG time series from the same ROI. The strictly causal multivariate autoregressive model (MVAR) that fit the data is of the form

$$
\begin{aligned}
& x(n)=\sum_{k=1}^{p} A(k) x(n-k)+\sum_{k=1}^{p} B(k) y(n-k)+u^{x}(n) \\
& y(n)=\sum_{k=1}^{p} A(k) y(n-k)+\sum_{k=1}^{p} B(k) x(n-k)+u^{y}(n)
\end{aligned}
$$

where $A(k)$ and $B(k)$ are the model co-efficient matrices, $p$ is the optimal model order chosen by Schwarz Bayesian Criterion (SBC) [18], [19], $n$ is the time index and $k$ is the 
lag. MVAR model parameter are estimated by the Kalman filter [20]. In addition, $u^{x}(n)$ and $u^{y}(n)$ are the additive white Gaussian noises' at a time $n$ for the time series $x$ and $y$ respectively. The wavelet transform of the considered time series $x$ is

$$
W^{x}(n, a)=\sqrt{\frac{\Delta t}{a}} \sum_{n^{\prime}=1}^{N} x_{n} \Psi_{o}^{*}\left[\left(n^{\prime}-n\right)\left(\frac{\Delta t}{a}\right)\right]
$$

similarly for the time series $y$ is

$$
W^{y}(n, a)=\sqrt{\frac{\Delta t}{a}} \sum_{n^{\prime}=1}^{N} y_{n} \Psi_{o}^{*}\left[\left(n^{\prime}-n\right)\left(\frac{\Delta t}{a}\right)\right]
$$

where $n$ is the time index and $a$ the wavelet scale. $N$ is the length of the corresponding time series $x$ and $y$ with sampled at equal time step size of $\Delta t$. The function $\Psi_{o}$ is selected to be the complex Morlet wavelet and expressed as

$$
\Psi_{o}(\eta)=\pi^{-\frac{1}{4}} e^{j w_{o} \eta} e^{-\frac{-\eta^{2}}{2}}
$$

and the cross wavelet transform can be defined for two timeseries as

$$
W^{x y}(n, a)=W^{x}(n, a) W^{y *}(n, a)
$$

The mathematical expression of the wavelet coherence is written as

$$
(C(n, a))^{2}=\frac{\left|W^{x y}(n, a)\right|^{2}}{W^{x x}(n, a) W^{y y}(n, a)}
$$

However, the major difference between Fourier and wavelet analysis are the selection of the window size. In the case of Fourier, the window size is fixed, whereas in wavelet it is adapted to the frequency of the signal. That is the reason; the wavelet transform has more precise time-frequency resolution than Fourier analysis. Many other tools are existing for time-frequency analysis and can be convenient to neurophysiologic signals. Nevertheless, the wavelet analysis offers both time-varying power spectrum and phase spectrum, which are desirable to compute coherence. Because of this, wavelet studies an obvious choice for the estimation of coherence amongst nonstationary signals. The wavelet-based estimation of coherence, or wavelet coherence, is a very recent tool. To our knowledge, it is only (briefly) mentioned in the papers cited above [5], [21], [22] which do not provide information crucial for its application to brain connectivity and their distinction.

Coherence can be defined as the frequency domain representation of correlation. The time-frequency precision trade off mostly recommended for the non-stationary signal such as EEG. Hence, the WC is mostly famous for measuring the synchrony in two timeseries data [23]. However, the EEG signal obtained from the human brain mostly 3D surface. Thus the impact and interact between two series are also affected by the presence of the third time series. The waveletbased coherence can be used to interpret the brain connectivity in different cognitive activity is described in the following subsection.

\section{Brain connectivity estimation using wavelet coherence} (WC)

Wavelet coherence is rarely used to investigate the cortical distinctiveness among different brain region involved in a cognitive task. The average value at a different frequency band of the wavelet coherence has been calculated based on the different ROI indicating distinct brain lobes. These average values are decomposed into brain spectra using DWT, and the WC of two signals can be calculated by using (3) yields a connectivity between the pair of electrodes or more than two electrodes. The WC based connectivity estimation is the quite advanced topic to be addressed. It is the time-frequencyspace by successively convolving the time series with scaled and translated versions of a wavelet function. This waveletbased coherence measurement is deliberately significant in the contribution of the functional brain connectivity and, using specifications that anticipate the brain activity involved in a particular cognitive action [24].

\section{RESULTS}

\section{A. Measuring Responses using Non-parametric Pairwise Co- herence}

The results obtained from the wavelet coherence (WC) has more or less distinctive however for the confirmatory test, we have opted for statistical distribution technique. The obtained results along with the error bar are shown in Fig. 3 and 4 for one of the meditator and non-meditator respectively.
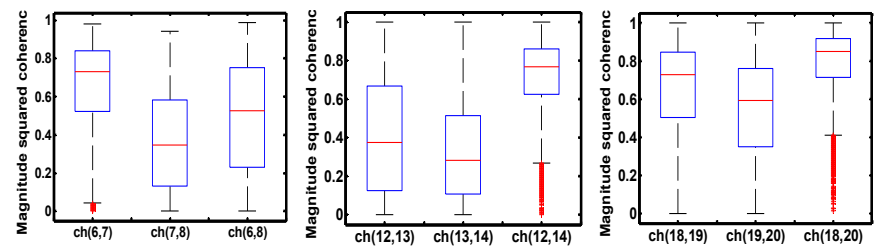

Fig. 3. Each box plot illustrates distribution percentiles of magnitude squared coherence between the channels pair mentioned. The bottom and top lines in each box depicts the 1 st and 3rd quartiles of the distribution; the band inside the box is the 2nd quartile. Wavelet Coherence in the selected ROI of EEG time series acquired during meditation of one of the meditator.
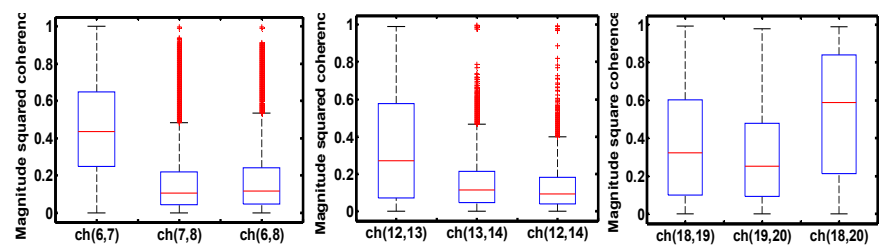

Fig. 4. Each box plot illustrates distribution percentiles of magnitude squared coherence between the channels pair mentioned. The bottom and top lines in each box depicts the 1st and 3rd quartiles of the distribution; the band inside the box is the 2nd quartile. Wavelet Coherence in the selected ROI of EEG time series acquired during resting state of one of the non-meditator.

The coherence connectivity is more inferable in the timefrequency domain than time or frequency domain alone. The primary constraint in EEG connectivity analysis is the detection of an actual connection between different brain regions 
TABLE I

ROI BASED WAVELET COHERENCE ESTIMATION IN MEDITATORS AND NON-MEDITATORS SUBJECTS AND THEIR RESPECTIVE BRAIN WAVE.

\begin{tabular}{|c|c|c|c|}
\hline $\begin{array}{l}\text { Selection of ROI and } \\
\text { channels based on } \\
\text { different } \\
\text { brain lobes }\end{array}$ & $\begin{array}{l}\text { Name of } \\
\text { the selected } \\
\text { channels as ROI }\end{array}$ & Meditators & Non-meditators \\
\hline 1. Prefrontal & Fp1, Fp2 & Alpha band & Beta band \\
\hline 2. Frontal & $\mathrm{F} 3, \mathrm{Fz}, \mathrm{F} 4, \mathrm{~F} 7, \mathrm{~F} 8$ & Delta band & Alpha band \\
\hline 3. Central & $\mathrm{C} 3, \mathrm{Cz}, \mathrm{C} 4$ & Theta band & Alpha band \\
\hline 4. Parietal & P3,Pz,P4,P7,P8 & Alpha band & Beta band \\
\hline 5. Occipital & $\mathrm{O} 1, \mathrm{O} 2$ & Delta band & Theta band \\
\hline $\begin{array}{l}\text { 6. Left and } \\
\text { Right temporal }\end{array}$ & $\mathrm{T} 7, \mathrm{~T} 8$ & Theta band & Beta band \\
\hline
\end{tabular}

and their inference out of the brain connectivity in a particular cognitive action such as meditation. The neural connectivity depends on the cause and effect of the neighbouring and nearby neurons. Here, in this paper, the connectivity estimation has been established in terms of EEG scalp electrodes. For the spatiotemporal analysis of the multichannel EEG, can be more visualized with respect to ROI based topographical representation.

\section{B. Topographical analysis and inferences based on WC con- nectivity}

The significance of this investigation was the evaluation of adopting EEG functional connectivity for the purpose of short term Kriya meditation. The topographical representation of magnitude of the wavelet coherence of one of ROI has been shown in Fig. 5 and 6 respectively. The average wavelet coherence and their corresponding wave bands are mentioned in Table I.
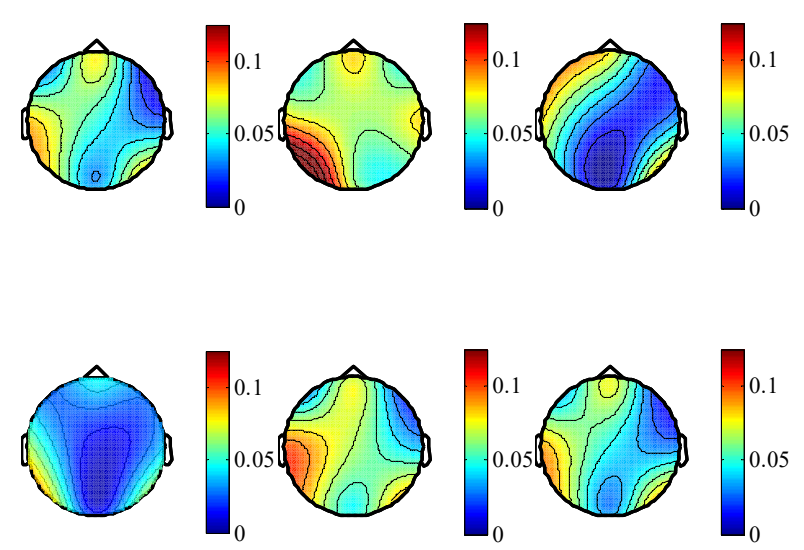

Fig. 5. Wavelet Coherence in the selected ROIs of EEG time series acquired during brain resting state of one of the non-meditator.

\section{DISCUSSION}

The WC was measured to observe functional connectivity in the brains of meditators and non-meditators. Moreover, the low frequency bands are affected by volume conduction which misleads to wrong interpretation of connectivity. WC results
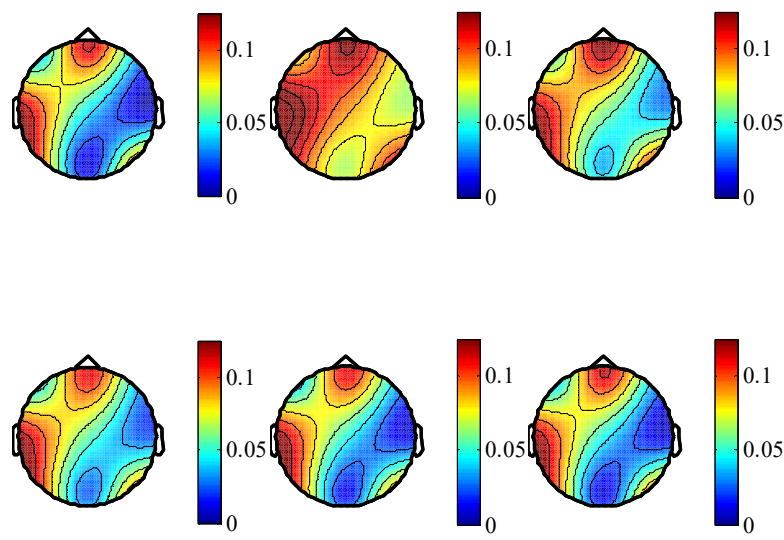

Fig. 6. Wavelet Coherence in the selected ROIs of EEG time series acquired during meditation of one of the meditator.

corresponds to delta $(\delta)$ and theta $(\theta)$ band can be discarded to avoid volume conduction. The decreased coherence represents the reduced functional connectivity between brain regions. It was observed faded WC in controlled group whereas increased WC was observed in meditator group in most channel pairs at most frequency bands. In specific, in meditator, increased WC was significantly observed in the pre-frontal region alpha $(\alpha)$ band (Table I). In the non-meditator or controlled group increased WC was primarily observed in the temporal-occipital links in the theta $(\theta)$ and beta bands $(\beta)$.

The human brains typically exhibit distinct dynamic behaviour at multiple spatial and temporal scale. Thus meditation EEG also has a distinct pattern in meditators and non-meditators. The topographical representation shows the synchronized oscillation among the EEG time series. The combination of WC and topographical approach are very rare study in the application of EEG involve in a cognitive task of short term meditation. The wavelet coherence shows the information about both time and frequency in EEG time series. The coherence study involves only two timeseries. If the study involves more than two timeseries then Granger causality and multivariate time-series analysis comes into picture.

\section{CONCLUSION}

In this conclusion, a unique distinction technique has been shown to classify two groups of subjects involved in the cognitive activity of meditation and resting state. Our approach has been proposed and analysed a novel way to categorize two groups i.e. meditators and non-meditators. Simultaneously it has also shown the time-varying connectivity between different brain regions. WC enabled us to examine the information flow during processing the cognitive task of meditation and resting state. Furthermore, the findings obtained from the proposed approach appears promising for their distinctive results using topographical results. The application to experimental data shows that the wavelet coherence offers different means of interdependence between brain signals as far as the detection of short-term coherence is concerned. The fact that these 
topographies did not occur in the control group strongly indicates that they are real features and with no artifacts. The different regions of the brain have the distinguishable degree of synchrony and information exchange patterns during meditation and resting brain state. The wavelet coherence can be considered as one of the unique features in the creation of the feature matrix for classification algorithms and can also be compared with other general linear model (GLM) based connectivity estimation which is deliberated as the extension of this work for future analysis.

\section{ACKNOWLEDGMENT}

The funds received from the Ministry of Human Resource Development, Government of India funded project 'CEHSandhi' is thankfully acknowledged. The authors would like to thank all the subjects for participation in the experiment and also thank to all members of the research team for their support for data collection.

\section{REFERENCES}

[1] S. Sanei and J. A. Chambers, EEG signal processing. John Wiley \& Sons, 2013.

[2] D. P. Subha, P. K. Joseph, R. Acharya, and C. M. Lim, "EEG signal analysis: a survey," Journal of medical systems, vol. 34, no. 2, pp. 195$212,2010$.

[3] S. L. Shapiro, J. A. Astin, S. R. Bishop, and M. Cordova, "Mindfulnessbased stress reduction for health care professionals: results from a randomized trial." International Journal of Stress Management, vol. 12, no. 2 , p. $164,2005$.

[4] A. Chiesa and A. Serretti, "Mindfulness-based stress reduction for stress management in healthy people: a review and meta-analysis," The journal of alternative and complementary medicine, vol. 15, no. 5, pp. 593-600, 2009.

[5] R. W. Thatcher, D. M. North, and C. J. Biver, "Development of cortical connections as measured by EEG coherence and phase delays," Human brain mapping, vol. 29, no. 12, pp. 1400-1415, 2008.

[6] W. Klonowski, W. Jernajczyk, K. Niedzielska, A. Rydz, and R. Stepien, "Quantitative measure of complexity of EEG signal dynamics," Acta Neurobiologiae Experimentalis, vol. 59, pp. 315-322, 1999.

[7] J.-P. Lachaux, A. Lutz, D. Rudrauf, D. Cosmelli, M. Le Van Quyen, J. Martinerie, and F. Varela, "Estimating the time-course of coherence between single-trial brain signals: an introduction to wavelet coherence," Neurophysiologie Clinique/Clinical Neurophysiology, vol. 32, no. 3, pp. 157-174, 2002.

[8] O. Scarpino, M. Guidi, and G. Bolcioni, "Topographic EEG analysis. methods for graphic representation and clinical applications." Acta neurologica, vol. 12, no. 5, pp. 410-426, 1990.

[9] W. R. Winter, P. L. Nunez, J. Ding, and R. Srinivasan, "Comparison of the effect of volume conduction on EEG coherence with the effect of field spread on MEG coherence," Statistics in medicine, vol. 26, no. 21, pp. 3946-3957, 2007.

[10] Z. Sankari and H. Adeli, "Probabilistic neural networks for diagnosis of alzheimer's disease using conventional and wavelet coherence," Journal of neuroscience methods, vol. 197, no. 1, pp. 165-170, 2011.

[11] D. La Rocca, P. Campisi, B. Vegso, P. Cserti, G. Kozmann, F. Babiloni, and F. D. V. Fallani, "Human brain distinctiveness based on EEG spectral coherence connectivity," IEEE Transactions on Biomedical Engineering, vol. 61, no. 9, pp. 2406-2412, 2014.

[12] X. Liu, H. Qi, S. Wang, and M. Wan, "Wavelet-based estimation of EEG coherence during chinese stroop task," Computers in biology and medicine, vol. 36, no. 12, pp. 1303-1315, 2006.

[13] R. Saab, M. McKeown, L. Myers, and R. Abu-Gharbieh, "A wavelet based approach for the detection of coupling in EEG signals," in Neural Engineering, 2005. Conference Proceedings. 2nd International IEEE EMBS Conference on. IEEE, 2005, pp. 616-620.
[14] L. Shaw and A. Routray, "Efficacy of adaptive directed transfer function for neural connectivity estimation of EEG signal during meditation," in Signal Processing and Integrated Networks (SPIN), 2015 2nd International Conference on. IEEE, 2015, pp. 198-202.

[15] L. Shaw, S. Mishra, and A. Routray, "Generalised orthogonal partial directed coherence as a measure of neural information flow during meditation," in Advancements of Medical Electronics. Springer, 2015, pp. $137-148$.

[16] C. S. Herrmann, M. Grigutsch, and N. A. Busch, "11 EEG oscillations and wavelet analysis," Event-related potentials: A methods handbook, p. 229, 2005.

[17] H. Adeli, Z. Zhou, and N. Dadmehr, "Analysis of EEG records in an epileptic patient using wavelet transform," Journal of neuroscience methods, vol. 123, no. 1, pp. 69-87, 2003.

[18] B. L. P. Cheung, R. Nowak, H. C. Lee, W. Drongelen, and B. D. Veen, "Cross validation for selection of cortical interaction models from scalp eeg or meg," IEEE Transactions on Biomedical Engineering, vol. 59, no. 2, pp. 504-514, 2012

[19] C. Porcaro, F. Zappasodi, P. M. Rossini, and F. Tecchio, "Choice of multivariate autoregressive model order affecting real network functional connectivity estimate," Clinical Neurophysiology, vol. 120, no. 2, pp. 436-448, 2009

[20] E. Möller, B. Schack, M. Arnold, and H. Witte, "Instantaneous multivariate eeg coherence analysis by means of adaptive high-dimensional autoregressive models," Journal of neuroscience methods, vol. 105, no. 2, pp. 143-158, 2001 .

[21] R. Coben, A. R. Clarke, W. Hudspeth, and R. J. Barry, "EEG power and coherence in autistic spectrum disorder," Clinical Neurophysiology, vol. 119 , no. 5, pp. 1002-1009, 2008.

[22] R. Srinivasan, P. L. Nunez, and R. B. Silberstein, "Spatial filtering and neocortical dynamics: estimates of EEG coherence," IEEE Transactions on Biomedical Engineering, vol. 45, no. 7, pp. 814-826, 1998.

[23] S. A. Markazi, S. A. Qazi, and L. Stergioulas, "Study of change blindness EEG synchronisation using wavelet coherence analysis," in Engineering in Medicine and Biology Society, 2005. IEEE-EMBS 2005. 27th Annual International Conference of the. IEEE, 2006, pp. 59955998.

[24] D.-H. Jeong, Y.-D. Kim, I.-U. Song, Y.-A. Chung, and J. Jeong, "Wavelet energy and wavelet coherence as EEG biomarkers for the diagnosis of parkinsons disease-related dementia and alzheimers disease," Entropy, vol. 18, no. 1, p. 8, 2015. 